FORMATION Formation emploi

Revue française de sciences sociales

98 | avril-juin 2007

Pour une approche par les capacités

\title{
Le travail et le développement des capacités
}

Work and the development of human capability

Human Capability Development und Arbeit

Jane Bryson et Kiri Merritt

\section{(2) OpenEdition}

Journals

Édition électronique

URL : http://journals.openedition.org/formationemploi/1777

DOI : 10.4000/formationemploi.1777

ISSN : 2107-0946

Éditeur

La Documentation française

Édition imprimée

Date de publication : 1 avril 2007

Pagination : 41-54

ISSN : 0759-6340

Référence électronique

Jane Bryson et Kiri Merritt, « Le travail et le développement des capacités », Formation emploi [En ligne], 98 | avril-juin 2007, mis en ligne le 30 juin 2009, consulté le 21 décembre 2020. URL : http:// journals.openedition.org/formationemploi/1777 ; DOI : https://doi.org/10.4000/formationemploi.1777

(c) Tous droits réservés 


\section{Numéro spécial}

\section{Le travail et le développement des capacités?}

Par Jane Bryson et Kiri Merritt*

\section{La qualité du travail joue un rôle important dans le développement des capabilités des salariés. À l'inverse, en Nouvelle-Zélande, de nombreux freins à ce développement sont apparus aux niveaux organisationnel et institutionnel.}

Le travail peut jouer un rôle critique dans le développement des capacités. Cet article vise à mieux comprendre le rôle du travail au travers d'une revue de la littérature récente consacrée aux capabilités et d'une discussion des conclusions de nos recherches portant sur le développement des capacités dans des organisations néo-zélandaises. Dans un premier temps, nous commentons les diverses théories relatives aux capabilités et nous mettons en évidence les points communs et les différences entre ces théories, puis nous les comparons à la notion plus large de capabilités exposée par l'économiste Amartya Sen. Dans un deuxième temps, nous commentons les conclusions des recherches concernant les moteurs, les éléments contributifs et les freins au développement des capacités. Nous abordons enfin l'interconnexion entre travail et capabilités et les questions que cela soulève.

${ }^{1}$ Le mot anglais «capability» est traduit par le néologisme « capabilité » ou, par souci de simplicité, par « capacité » dans les expressions « approche par les capacités » et « développement des capacités ».

Ces travaux de recherche ont été rendus possibles grâce aux fonds de la New Zealand Foundation for Research, Science \& Technology alloués au projet « Développement des capacités humaines : institutions de l'emploi, organisations et individus ».
* Jane Bryson est psychologue organisationnelle, maître de conférence à la Management School, université Victoria de Wellington, Nouvelle-Zélande. Elle est également l'une des deux responsables d'un projet de recherche intitulé "Développement des capacités humaines: institutions de l'emploi, organisations et individus », qui explore les différentes influences sur le développement des capacités au sein d'organisations néo-zélandaises. Ses travaux portent principalement sur la gestion des ressources humaines, les capacités organisationnelles et individuelles, et ont trait aux professions libérales, aux cadres dirigeants et aux changements professionnels. jane.bryson@vuw.ac.nz ou tél. : + NZ 6444635707.

Kiri Merritt est chargée de recherche Maori à l'université Victoria de Wellington, Nouvelle-Zélande. Elle est titulaire d'un master en psychologie de l'université Waikato, Hamilton, Nouvelle-Zélande. Elle a travaillé précédemment comme chargée de recherche sur le projet « Développement des capacités humaines : institutions de l'emploi, organisations et individus». Elle poursuit des recherches sur le développement de carrière de la population indigène maori. Ses domaines de recherche ont trait au développement des carrières, au développement du personnel et à l'éducation des Maoris. 
La littérature consacrée aux capabilités est abondante et recouvre une diversité de disciplines allant de l'économie à l'éducation, de la gestion à la politique publique. Chaque discipline attribue des sens et des usages différents au terme " capabilité » en fonction de sa nature. Dans la brève discussion ci-dessous, plusieurs facteurs communs émergent : l'importance du développement des individus, le rôle significatif que le travail peut jouer et la portée de l'interaction entre l'individu et l'organisation en matière de développement des capacités. Des points faibles communs se dégagent également : une tendance à ne pas placer l'individu au centre de tout et, en particulier, l'impact des structures institutionnelles, des politiques organisationnelles et du comportement des individus sur le développement et l'expression des capacités.

\section{CAPABILITÉS ET TRAVAIL}

Ce chapitre présente deux grands courants de pensée sur les capabilités. L'approche par les capacités de Sen, d'une part, et les capabilités telles que définies dans la littérature consacrée à la gestion et l'organisation, d'autre part.

\section{Développement et bien-être selon A. Sen}

Dans cette partie, nous décrivons l'approche par les capacités de Sen. Nous étudions en particulier la manière dont le travail peut s'inscrire dans cette notion de capabilités. Sen (1999) a remis en question la vision purement économique du développement, uniquement perçu en termes d'augmentation du PIB (produit intérieur brut) ou du revenu réel, au profit d'une vision plus large du développement qui intègre les opportunités et l'espace de liberté dont dispose un individu pour mener la vie choisie. Dans sa forme la plus simple, l'approche par les capacités définit le bien-être comme « la capacité d'un individu à être et faire ce à quoi il aspire» (Sen, 1993, p. 30). L'approche de Sen se fonde sur les notions de fonctionnements et de capabilités. Les fonctionnements sont définis comme "tout ce qu'il est possible de faire dans la vie, toutes les façons d'être et d'agir des individus » (p. 31). Il n'existe pas de liste définitive des fonctionnements mais on peut citer par exemple le fait d'avoir un toit ou un emploi rémunéré. Dès lors, le concept de capabilité «reflète les diverses combinaisons de fonctionnements qu'un individu peut accomplir, et parmi lesquelles il peut faire son choix» (p. 31). Le développement des capacités s'appuie donc sur les fonctionnements et nécessite une reconnaissance des opportunités, des libertés et des avantages (Basu et Lopez-Calva, 2002). L'évaluation de la qualité de vie peut résulter de la capacité d'un individu à pouvoir effectivement réaliser les fonctionnements auxquels il accorde de la valeur (Sen, 1993).

Dans ses travaux, Sen n'établit aucune liste des capabilités fondamentales. En revanche, Nussbaum (1999) a dressé, dans le cadre d'une approche par les capacités, une liste de capabilités fonctionnelles de base, indispensables à un «fonctionnement véritablement humain ». Elle répertorie onze capabilités fonctionnelles de base, depuis «Pouvoir vivre, autant que possible, une vie humaine complète jusqu'à la fin » jusqu'à "Pouvoir vivre notre propre vie dans un environnement et un contexte de notre choix. » Cette dernière inclut le droit à un emploi selon le principe d'égalité et le droit de travailler dans la dignité.

De récentes études ont permis de mieux comprendre le travail et sa contribution à l'approche par les capacités. Par exemple, Burchardt (2002) a soulevé la question de la capabilité d'emploi des femmes et ses recherches ont démontré que de nombreuses femmes qui n'ont pas d'emploi rémunéré manquent de capabilité d'emploi. Elle suggère que la capabilité d'emploi est une mesure plus utile que des mesures telles que le taux de chômage. Salais et Villeneuve (2004) revendiquent l'utilisation de l'approche par les capacités comme moyen de concilier les objectifs sociaux et économiques au niveau institutionnel. Ils affirment que la «capabilité se développe (ou diminue) en fonction des conditions de vie et de travail quotidiennes» (p. 12) et que cet aspect est essentiel dans le traitement de problématiques « à la fois individuelles et collectives couvrant l'employabilité, la formation tout au long de la vie, la prévention des risques économiques et sociaux, le juste et libre équilibre entre vie professionnelle et vie privée, 
la sécurité sociale active» (p. 16). Enfin, Sehnbruch (2004) a appliqué l'approche par les capacités au marché du travail et a suggéré qu'une telle approche exige de prendre en compte la qualité de l'emploi au lieu de se concentrer uniquement sur les taux d'emploi ou de chômage.

La vie de nombreuses personnes est en grande partie absorbée par le travail. Il est donc primordial que nous essayions de comprendre en quoi le travail peut constituer un moteur ou un frein, selon la notion de capabilités de Sen. Le travail peut répondre à différents besoins d'un individu et contribuer ainsi à son bien-être. Selon Burchardt (2002), un emploi rémunéré constitue une contribution significative au revenu, à l'estime de soi et à l'interaction sociale, outre sa valeur en tant qu'activité. Dans d'autres études (Deeks et Rasmussen, 2002), le travail a été défini comme un moyen en vue d'une fin (le travail comme moyen de survivre, de financer des objectifs de vie extra-professionnels, de servir la communauté) ou comme une fin en soi (le travail procure une satisfaction intrinsèque basée sur la croissance psychologique, la créativité, le statut, la progression de carrière). Le travail peut donc utiliser les fonctionnements et les capabilités des individus et leur permettre d'apporter leur contribution à l'organisation, de se sentir compétents professionnellement, de renforcer la confiance en leurs capacités, de s'engager dans une activité utile, d'être un membre reconnu de la société et de groupes sur son lieu de travail, d'obtenir des ressources qui leur serviront à entretenir d'autres personnes et à mener la vie qu'ils souhaitent. Voici, pour l'essentiel, ce que le travail peut apporter aux individus en termes de capabilités.

Mais on sait également qu'un travail peut déresponsabiliser des salariés, voire les exploiter, qu'il existe des employeurs versatiles et plus soucieux d'atteindre leurs objectifs financiers que de promouvoir le bien-être des salariés. C'est pourquoi les politiques publiques et la législation sur l'emploi s'efforcent d'offrir des protections institutionnalisées aux salariés et aux employeurs. Elles établissent les règles de base régissant la relation d'emploi et l'expérience de travail.

Le travail peut jouer un rôle fondamental dans la capacité des individus à mener la vie qu'ils ont choisie. La relation d'emploi et la législation qui la régit constituent un élément central de la notion de capabilités de Sen. Le travail peut représenter un frein ou un facteur du bien-être des individus.

\section{Les autres visions de la capabilité appliquée au travail}

Dans cette partie, nous passons brièvement en revue les différents concepts de capacités dans la littérature consacrée à la gestion. Nous comparons également ces concepts avec la vision de Sen. La littérature sur la gestion et l'organisation pourrait laisser croire à une vision unique du concept de capacités, alors qu'il n'en est rien (Brown et McCartney, 2004). Divers courants de pensée sur la nature et la fonction des capacités sont représentés dans cette littérature, allant des approches les plus restrictives, focalisées sur les avantages pour l'organisation (par exemple dans la littérature consacrée à la gestion stratégique), à des approches plus holistiques qui considèrent le développement conjoint de l'organisation et de l'individu (par exemple dans la littérature consacrée au développement de l'organisation). L'usage et la définition du terme « capabilité » sont souvent déterminés par le résultat recherché. Néanmoins, cette littérature se caractérise généralement par un utilitarisme étroit des concepts de capacités. Des notions plus larges, telles que l'approche par les capacités de Sen, y sont rarement abordées et lorsque c'est le cas, par exemple dans certaines théories du développement de l'organisation et de l'organisation apprenante qui reconnaissent le potentiel humain, elles servent en fin de compte les intérêts de l'organisation. Ainsi, la littérature sur la gestion et l'organisation a tendance à mesurer les capacités à l'aune des performances des salariés et des augmentations de productivité qui devraient en résulter.

Une branche reconnue de cette littérature suggère que la productivité et la performance organisationnelles résultent essentiellement d'une augmentation des compétences et des connaissances des salariés (Pfeffer, 1994 ; Huselid, 1995 ; Becker et Gerhart, 1996). La théorie du capital humain sous-tend nombre de ces hypothèses dans des disciplines liées à la gestion et à l'organisation. À cet égard, la distinction entre la théorie du capital humain et la théorie des capacités est révélatrice. Selon Sen (1997, p. 1959), le capital humain se réfère à « la capacité d'individus - 
à travers leurs compétences, leurs connaissances et leurs efforts - d'augmenter les possibilités de production ». En revanche, la capabilité est définie comme la «capacité d'individus à mener la vie qu'ils ont des raisons de valoriser » (Sen, 1997 p. 1959). Sen commente la nature des deux concepts et présente quelques points de comparaison importants. Tout d'abord, les deux concepts sont centrés sur les individus et leurs capacités et ont donc, à cet égard, un point commun. Ils présentent toutefois des différences : en effet, le capital humain est souvent perçu en termes de contribution à la productivité de l'organisation, tandis que la capabilité est considérée comme une contribution plus large permettant aux individus d'améliorer leur vie de façon générale. En fait, nous retrouvons ici la même distinction qui existe entre employeurs et salariés. Les employeurs veulent développer les capacités des salariés afin d'améliorer la production, tandis que les salariés développent leurs capacités non seulement pour le travail, mais également pour contribuer à leur bien-être général.

Dans une certaine mesure, la capabilité est définie différemment selon que vous percevez la capabilité individuelle comme un objectif final, ou que vous considérez l'individu comme un moyen d'atteindre les objectifs organisationnels.

Les approches du capital humain ont permis de mieux cerner la relation entre travail et apprentissage. Une branche très influente de cette littérature est le concept d'organisation apprenante présenté par Senge (1990). Selon lui, en mettant en place certaines pratiques, une organisation peut devenir un lieu où les individus apprennent continuellement ensemble et développent leurs capacités afin de générer des résultats. Toutefois, le concept d'organisation apprenante peut susciter certaines critiques. D'aucuns avancent que le concept conduit à une forme de contrôle social et d'exploitation accrue des salariés, en particulier des femmes (Mojab et Gorman, 2003). D'autres prétendent qu'il n'est pas démontré que ce processus d'apprentissage apporte des avantages mutuels aux organisations et aux individus (Thomson, Mabey, Storey, Gray et Isles, 2001 ; Fenwick, 1998) :

«Selon le discours officiel, l'objectif de l'organisation apprenante est une libération et une responsabilisation des individus, mais cette libération est un piège: on promet l'émancipation aux individus en récompense de leur participation active à une organisation apprenante pour optimiser leur potentiel de salariés novateurs et intelligents - à condition que cet apprentissage serve la prospérité de l'organisation. » (Fenwick, 1998)

Certains auteurs ont préconisé aux organisations d'adopter une approche plus holitistique de l'apprentissage et du développement. Selon cette approche, le lieu de travail ne doit pas être considéré uniquement comme un environnement physique mais doit englober «le lieu physique, des idées et des objectifs communs, des comportements et des attitudes qui déterminent l'environnement de travail et les relations de travail » (Matthews, 1999, p. 19). Matthews défend une définition de l'organisation apprenante qui privilégie la réalisation à la fois des objectifs individuels et organisationnels.

Dans la même veine, la littérature sur les relations professionnelles (à savoir les relations entre employeurs et salariés) propose un contrepoint aux visions exposées dans la littérature sur la gestion et l'organisation. Selon Peirce (2000), les relations professionnelles doivent trouver un équilibre entre efficacité et équité et prendre en compte les points de vue des salariés et du patronat. Plus récemment, Budd (2004) a suggéré que la voix des salariés soit prise en considération dans la relation d'emploi, et ce dans un souci d'équité et d'efficacité.

Les arguments ayant trait aux relations professionnelles se fondent sur l'hypothèse d'un déséquilibre de pouvoir entre l'employeur et le salarié. Afin d'équilibrer les intérêts de l'employeur et du salarié, il convient de privilégier les recours institutionnels qui permettent aux salariés de développer leurs capacités et de s'épanouir, tout en servant les intérêts de l'employeur. Les droits et les protections en matière d'emploi constituent un recours institutionnel important qui se traduit par divers mécanismes législatifs. Les syndicats constituent un autre recours de poids. Ils peuvent en effet représenter les salariés et leur permettre d'influencer leur environnement de travail. Cependant, au niveau macro-économique, la législation en matière d'emploi exerce une influence notable sur le rôle des syndicats. Ainsi, en Nouvelle-Zélande, l'Employment Contracts Act 1991 a fortement contribué au déclin considérable des négociations 
collectives et du nombre de syndiqués dans les années 90. Cette loi a restreint l'activité des syndicats et l'accès des salariés aux syndicats et a rendu les négociations collectives plus difficiles (Deeks et Rasmussen, 2002). Plus récemment, la NouvelleZélande a adopté l'Employment Relations Act 2000 qui offre une meilleure protection de l'activité syndicale, encourageant à la fois les négociations collectives et l'adhésion des salariés aux syndicats (Blackwood, Feinberg-Danieli, Lafferty et Kiely, 2005). L'environnement institutionnel et politique subit des changements. Cela peut avoir de fortes répercussions sur l'aptitude des salariés à protéger leur propre bien-être et celui des autres salariés.

Le développement des capacités est une question controversée qui requiert une attention sérieuse et soutenue compte tenu de son impact sur les individus, les organisations et les sociétés. Notre brève analyse de la littérature a montré l'existence d'un large éventail de concepts en matière de capacités, allant de versions plus restrictives, associées à des disciplines telles que la gestion et l'organisation, à des notions plus larges résumées dans les débats sur l'approche par les capacités de Sen. Les principales différences entre ces approches résident dans les résultats attendus du développement des capacités. Le point commun de ces approches est que, indépendamment du résultat recherché, les fonctionnements et les capabilités des individus, leur capacité à mener la vie de leur choix, sont influencés par leurs expériences quotidiennes au travail.

Pour étudier le rôle du travail dans le développement des capacités, il convient de prendre en considération des notions plus larges des capabilités. Circonscrire la discussion et le débat dans des perspectives restrictives prenant uniquement en compte les objectifs organisationnels est révélateur d'une incapacité à replacer le travail dans le contexte de la vie des individus et à appréhender les dimensions sociales et économiques associées au travail. Nous devons également chercher à comprendre, sous le prisme de notions plus larges des capacités, telles que prônées par Sen et d'autres, en quoi le travail peut constituer un frein ou être un moteur du développement des capacités. Tant que ces liens ne seront pas établis, les politiques institutionnelles et les pratiques organisationnelles continueront à être guidées par des recherches et des études dont les arguments sont fondés sur des visions étroites des capacités, notamment celles servant les seuls intérêts des employeurs.

\section{LE DÉVELOPPEMENT DES CAPACITÉS AU TRAVAIL}

Cette partie présente une recherche empirique utilisant l'approche par les capacités. Elle décrit les méthodes de recherche utilisées, la base de sondage et le cadre analytique appliqué aux données.

La recherche a pour objet d'identifier et d'étudier l'influence que les individus, les organisations et les institutions exercent les uns sur les autres en matière de développement des capacités. Les organisations et les institutions constituent le cadre dans lequel les individus fonctionnent; dès lors, elles déterminent la liberté de choix et d'action des individus par rapport à leur propre développement. Nous nous appuyons sur un vaste programme de recherche appliquée réalisé à l'université Victoria de Wellington, intitulé « Développement des capacités humaines: institutions de l'emploi, organisations et individus ». Ce programme étudie l'impact des facteurs institutionnels, organisationnels et individuels sur le développement des capacités dans des organisations néo-zélandaises.

Le projet a utilisé une approche multiniveaux en introduisant la notion d'ensemble de capacités dans la recherche empirique. Cela signifie que la recherche porte sur les capacités individuelles dans le contexte élargi d'un réseau de connexions entre organisation, industrie et région géographique, autant de facteurs qui influencent le développement des capacités. Les études de cas ont donc impliqué des discussions avec des dirigeants, des salariés et des syndicats, ainsi que la collecte d'informations auprès d'organes externes intervenant, au niveau des régions et des industries, dans le développement des capacités (par exemple les prestataires de formation, les organes professionnels, etc.). Par ailleurs, lors des entretiens individuels, nous avons demandé aux salariés de réfléchir à l'historique du développement de leurs capacités, aussi bien dans leur environnement familial que scolaire et professionnel, afin de dessiner une image précise des moteurs et des freins 
au développement. Nous pensons que cette approche permet une meilleure compréhension du contexte global du développement des capacités.

Selon Yin (2003), l'objet des études de cas est d'élaborer une théorie, complétant ou confirmant probablement des explications particulières issues de catégories générales, en l'occurrence ici la théorie du développement des capacités au travail. Dans ce projet, les études de cas contribueront à élaborer un cadre permettant de comprendre le développement des capacités dans le contexte d'organisations néozélandaises. La conception globale de nos études de cas combine une approche collective et stratifiée (Stake, 1995 ; Patton, 2002). Autrement dit, elle s'appuie sur plusieurs cas dans un souci de mieux appréhender les questions de recherche. À travers ce processus de stratification, nous étudions des cas individuels tout en explorant des schémas communs aux différents cas.

\section{Encadré 1 \\ Des entretiens semi-structurés selon l'approche par les capacités}

La principale méthode utilisée reposait sur des entretiens semi-structurés. L'objectif étant de savoir comment les personnes percevaient le développement de leurs capacités au travail et les opportunités de développement, les entretiens ont été considérés comme la méthode la mieux appropriée. Les entretiens étaient approfondis et semi-structurés (Kvale, 1996). Un scénario d'entretien flexible a été utilisé, puis complété et modifié tout au long de la période d'entretiens. Les frontières délimitant les sujets susceptibles d'êtres explorés n'étaient aucunement figées, et les entretiens étaient destinés à traiter des questions à la fois individuelles, organisationnelles et institutionnelles. Les entretiens avec les salariés comportaient les questions générales suivantes : comment avez-vous développé vos compétences et vos capacités tout au long de votre vie professionnelle et au sein de votre organisation actuelle ? Quelles ont été les opportunités de formation et de développement et comment y avez-vous réagi ? Quels sont vos projets professionnels et vos projets de développement des capacités pour l'avenir et quels facteurs ont favorisé ou desservi le développement de vos capacités individuelles? Les entretiens avec des dirigeants et des propriétaires ont couvert les mêmes questions que celles posées aux salariés, avec en plus une question sur la manière dont ils développaient les compétences de leurs collaborateurs et dont l'organisation et l'industrie en général appréhendaient le développement des compétences et des capacités. Les entretiens avec des prestataires de formation et des représentants de l'industrie portaient sur les pratiques et les enjeux du développement des capacités (moteurs et freins) pour l'industrie.

Des entretiens ont été menés avec le personnel à tous les niveaux. Tous les entretiens ont été enregistrés et transcrits. L'analyse a été réalisée selon la méthode des gabarits (King, 1998) assistée par le logiciel N6. Pour le présent article, des codes ont été conçus afin de classer les commentaires sur le développement des capacités dans les catégories suivantes : 1) les moteurs ; 2) les éléments contributifs; 3) les freins au développement des capacités. Les moteurs sont définis comme les facteurs qui catalysent activement le développement des capacités. Les éléments contributifs sont les facteurs qui soutiennent le développement des capacités mais qui sont incapables de déclencher un processus de développement sans la présence d'un moteur. Les freins se réfèrent aux facteurs qui empêchent les individus de se développer et/ou d'exploiter leurs capacités au travail. Les freins peuvent être tout simplement l'absence de moteurs, mais il existe également des freins actifs qui empêchent le développement des capacités, même en présence de moteurs. Ces trois catégories ont ensuite été divisées en sous-catégories de facteurs: 1) les facteurs individuels; 2) organisationnels, liés aux pratiques en vigueur dans une organisation ; 3) institutionnels et liés à des dispositifs systémiques à l'échelle de la société, tels que la politique, la législation, les réglementations et les attitudes sociales liées à l'éducation, l'emploi, le développement industriel et l'économie. Deux membres de l'équipe de recherche ont classé indépendamment les données dans ce cadre, puis ont déterminé ensemble les catégorisations. 
Dans cet article, notre analyse s'appuie sur une étude de cas pilote chez un fabricant de produits de consommation courante et sur deux études de cas dans l'industrie du vin et l'industrie de fabrication de meubles. Ces études de cas représentent au total 100 entretiens, dans 20 organisations différentes ${ }^{2}$.

\section{Les principaux facteurs et freins au développement des capacités au travail}

Cette partie présente les conclusions de notre recherche empirique qui a exploré les facteurs influençant le développement des capacités au travail en Nouvelle-Zélande ${ }^{3}$. Les principaux facteurs qui ressortent de notre analyse des transcriptions d'entretiens sont synthétisés dans le Tableau 1.

\footnotetext{
2 Bien que non citées dans cet article, d'autres études sont en cours, et notamment sur les approches du développement des capacités dans des organisations maori (les Maoris sont les populations indigènes de Nouvelle-Zélande).

${ }^{3}$ Il convient de souligner que la moitié des salariés du secteur privé en Nouvelle-Zélande sont employés dans de petites et moyennes entreprises (ministère du Développement économique, 2003). Aucune loi ou réglementation n'oblige les employeurs à former ou développer les compétences de leur personnel. L'enseignement secondaire est obligatoire jusqu'à 16 ans.
}

\section{Les facteurs individuels et organisationnels, moteurs des capacités}

Les moteurs du développement des capacités, dans ces organisations néo-zélandaises, ont été identifiés uniquement au niveau de l'individu et de l'organisation. Les facteurs individuels étaient essentiellement liés à "l'attitude», ce qui n'a rien d'étonnant. En tant que moteur, l'attitude a été décrite comme un «état d'esprit positif», la volonté « d'entreprendre des choses » ou de « sortir de sa zone de confort ». Elle a également été caractérisée comme l'intérêt qu'une personne porte à ce qu'elle fait et donc le désir de se montrer à la hauteur et d'apprendre. Outre l'attitude, trois autres moteurs individuels ont été fréquemment cités : l'aspiration à la réussite, la pro-activité et des priorités non liées au travail.

L'aspiration à la réussite pour soi-même ou pour ses proches constituait un moteur important pour de nombreux salariés. Des salariés immigrants et des salariés néo-zélandais de souche, issus de milieux socio-économiques plus défavorisés, ont indiqué qu'ils développaient leurs capacités afin d'offrir à leurs enfants une vie meilleure. Quelques salariés plus jeunes, sans responsabilités familiales, ont

Tableau 1

Les principaux facteurs de développement des capacités

\begin{tabular}{|c|c|c|c|}
\hline & Moteurs & Éléments contributifs & Freins \\
\hline Institutionnels & $\begin{array}{l}\text { - Attitudes sociales } \\
\text { - Infrastructure de formation } \\
\text { en entreprise }\end{array}$ & $\begin{array}{l}\text { - État de l'industrie } \\
\text { et de l'économie }\end{array}$ & $\begin{array}{l}\text { - Attitudes sociales } \\
\text { - Infrastructure de formation } \\
\text { en entreprise/politique } \\
\text { - Influences de l'école } \\
\text { sur le choix du métier }\end{array}$ \\
\hline Organisationnels & $\begin{array}{l}\text { - Soutien et encouragement } \\
\text { des employeurs et supérieurs } \\
\text { hiérarchiques } \\
\text { - Salaire } \\
\text { - Conception et pratiques } \\
\text { de travail }\end{array}$ & $\begin{array}{l}\text { - Avantages sociaux } \\
\text { ou autres des salariés } \\
\text { - Bonnes relations avec } \\
\text { les clients et les fournisseurs }\end{array}$ & $\begin{array}{l}\text { - Vision à court terme des } \\
\text { dirigeants d'entreprise } \\
\text { - Organisations de petite taille } \\
\text { - Refus et réticence de parta- } \\
\text { ger savoir-faire et capacités } \\
\text { (entre les individus } \\
\text { et les organisations) }\end{array}$ \\
\hline Individuels & $\begin{array}{l}\text { - Attitude } \\
\text { - Aspiration } \\
\text { - Pro-activité } \\
\text { - Autres priorités }\end{array}$ & $\begin{array}{l}\text { - Aimer son travail } \\
\text { - Aptitude à apprendre }\end{array}$ & $\begin{array}{l}\text { - Attitude } \\
\text { - Manque de maîtrise } \\
\text { de la lecture et de l'écriture }\end{array}$ \\
\hline
\end{tabular}


déclaré vouloir réussir pour faire plaisir à leurs parents ou à une autre personne importante dans leur vie.

Nombre de nos analyses ont également mis en évidence un autre moteur significatif : la pro-activité. L'offre et l'exploitation d'opportunités de développement étaient positivement influencées par un comportement individuel proactif : être à l'initiative de la demande, ne pas être avare de son temps et façonner l'environnement de travail en fonction de ses besoins; dans ce cas là, on était susceptible d'obtenir le développement désiré.

Toutefois, nous avons également observé que chez certaines personnes, les «fonctionnements de valeur » se situent exclusivement hors de la sphère $\mathrm{du}$ travail. Par exemple, plusieurs personnes ont indiqué que leurs engagements familiaux ou la quête d'un autre mode de vie absorbaient leur temps libre et leur énergie. Certaines ont affirmé ne pas vouloir $\mathrm{du}$ surcroit de stress qui accompagne une promotion professionnelle. Parmi les salariés les plus jeunes, nous avons également observé une volonté de maximiser la rémunération à court terme avec un minimum d'efforts (par exemple, en changeant d'employeur au lieu de s'impliquer dans des activités de développement) pour profiter des loisirs. Pour beaucoup donc, le développement des capacités relevait de priorités non liées au travail. Cette observation s'inscrit davantage dans une perspective de capabilités que dans une vision du capital humain.

Trois principaux moteurs du développement des capacités ont émergé au niveau organisationnel : le salaire ; le soutien et l'encouragement de la part des dirigeants et supérieurs hiérarchiques et la définition même du travail. Quelques-unes des organisations étudiées rémunéraient leurs salariés sur la base des compétences et de la productivité. Dans de nombreux cas, le système de rémunération basé sur les compétences a été mis en place sous la pression de syndicats. Les salariés ont indiqué qu'un tel système était très incitatif en matière de développement des compétences. Toutefois, plusieurs salariés ont fait remarquer que même si l'acquisition de compétences conduisait à une augmentation de salaire, l'opportunité de mettre en pratique ces nouvelles capacités était souvent restreinte par la définition du poste ou la nature du travail.
D'un autre côté, la nature et la conception du travail ont été fréquemment citées comme facteurs de développement des capacités. La possibilité de réaliser des tâches différentes, un travail conçu de manière à englober un processus entier plutôt qu'un processus fragmenté en de multiples activités séparées, et la possibilité de mesurer (constater) le résultat de ses efforts, étaient considérés comme des facteurs importants du développement des capacités au travail.

Durant les entretiens, et cela mérite d'être souligné, beaucoup de salariés ont tenu à raconter des expériences de développement des capacités survenues grâce aux efforts de certains employeurs, dirigeants ou supérieurs hiérarchiques. Les salariés ont cité des employeurs qui prenaient des apprentis ou des stagiaires et leur offraient des opportunités de renforcer leurs compétences. Ils ont également évoqué en termes positifs ces employeurs qui « sortent des sentiers battus » et qui sont prêts à essayer de nouvelles méthodes de travail afin d'améliorer les capacités de l'organisation et de ses salariés. Dans toutes les industries étudiées, le principal moteur de développement était lié aux encouragements réguliers, au soutien et au coaching de dirigeants et supérieurs hiérarchiques qui permettaient de renforcer la confiance et les capacités des salariés. Pour certains salariés, une personne-clé, par exemple la «maman», un ami respecté ou un collègue, les avait encouragés. Cette personne-clé, qui n'était pas en mesure de leur offrir des opportunités ou un coaching, les aidait cependant à avoir confiance en eux, ce qui leur avait permis d'être plus proactifs dans la quête d'opportunités de développement de leurs capacités.

$\mathrm{Au}$ niveau institutionnel, les infrastructures de formation en entreprise (en Nouvelle-Zélande, elles ont vu le jour dans le cadre de l'Industry Training $A c t, 1992)$ sont jugées aptes à favoriser le développement des compétences, souvent assimilé au développement des capacités. Plus particulièrement, la réintroduction d'un système d'apprentissage (en 1999, après une absence de presque une décennie) a encouragé les personnes et les entreprises à investir davantage dans le développement des capacités. Par ailleurs, la création de postes de conseillers professionnels itinérants est citée comme un facteur de développement des capacités car ils ont aidé les 
entreprises à mieux soutenir leurs apprentis/ stagiaires. Ces conseillers visitent les lieux de travail afin d'évaluer les progrès des apprentis/stagiaires par rapport à des normes de qualification spécifiques à l'industrie concernée et ils s'assurent que ceux-ci acquièrent l'expérience nécessaire. Cette aide est encore plus précieuse pour les nombreuses petites entreprises, car les conseillers apportent un soutien à l'employeur et gèrent l'ensemble des documents administratifs relatifs aux apprentis/stagiaires; ils contribuent ainsi à lever en partie un frein important pour les petites entreprises qui ne souhaitaient pas ou n'étaient pas capables de gérer la paperasserie administrative liée à la formation.

Cependant, au niveau institutionnel, les attitudes sociétales face au travail, aux compétences et à l'éducation constituent à la fois un moteur et un frein. L'étude a mis en évidence une vision mitigée de la valeur que la société accorde à l'éducation. Les attitudes face aux différents types de travail sont influencées par les programmes scolaires, l'image de l'industrie et l'orientation de la politique gouvernementale.

Les éléments contributifs du développement des capacités étaient liés à des circonstances ou des caractéristiques importantes mais qui ne suffisaient pas, à elles seules, à induire le développement des capacités. Au niveau institutionnel, ces éléments contributifs englobaient l'état général de l'économie et de l'industrie concernée. Au niveau organisationnel, l'environnement de travail constituait un élément contributif manifeste; par exemple, proposer des activités sociales ou un environnement plus favorable au personnel conduisait à une "relative augmentation de la productivité en raison d'un léger changement d'attitude». Il pouvait s'agir de quelque chose d'aussi simple que de donner aux salariés le sentiment d'appartenir à l'organisation ou au groupe et d'être appréciés par leur employeur. Au niveau individuel, l'aptitude à apprendre contribuait au développement des capacités, au même titre qu'aimer son travail, «vous devez aimer ce que vous faites. Cela se reflète dans votre attitude, votre productivité - moins de corrections et moins d'erreurs » (extrait d'un entretien auprès d'un salarié d'une usine de fabrication de meubles).

\section{L'organisation et les institutions freinent les capacités}

Les freins au développement des capacités au travail sont apparus nombreux aux niveaux organisationnel et institutionnel. En revanche, au niveau individuel, les principaux freins identifiés étaient l'attitude et le manque de maîtrise de la lecture et de l'écriture. Parmi les attitudes constituant un frein au développement des capacités figuraient le fait de ne pas être intéressé par son travail, de refuser d'essayer et la paresse. Ces attitudes pouvaient être liées à un manque de maturité, de confiance en soi et à ce que certains prestataires de formation dans nos entretiens décrivaient comme une "peur de l'éducation ou de l'engagement dans un choix de carrière ». Peut-être que ces attitudes peuvent également être fortement liées au frein induit par le manque de maîtrise de la lecture et de l'écriture. En effet, des travailleurs immigrés mais également des travailleurs nés et éduqués en Nouvelle-Zélande possédaient une faible maîtrise de la lecture et de l'écriture. Par exemple, beaucoup ont déclaré fuir les opportunités de développement parce que: «je suis dyslexique», "l'école n'a jamais été mon truc», "je suis paresseux», «je suis trop vieux pour ça», "je n'aime pas les livres», "je ne comprends pas ce $q u$ 'on me dit». Dans cette catégorie, de nombreux salariés ont indiqué que de mauvaises expériences à l'école, des problèmes familiaux ou d'autres problèmes avaient perturbé leur scolarité et étaient la cause de leur faible niveau scolaire. Il semble qu'un mauvais niveau scolaire ait un impact, par la suite, sur la confiance et la volonté d'apprendre dans certains types de contexte.

Les plus grands freins organisationnels que nous avons observés et qui ont été cités étaient principalement liés à la petite taille des organisations et, plus important encore, à la vision étroite et à court terme des entreprises et des industries en matière de développement des capacités. Certaines organisations ont déclaré investir dans la formation ou dans toute autre forme de développement des capacités uniquement lorsqu'elles y étaient contraintes par la loi (par exemple, la formation à la santé et à la sécurité). Les petites entreprises n'avaient qu'un seul objectif: assurer leur survie grâce à la productivité de la maind'œuvre, mais avec un investissement minimum dans 
le développement des capacités. Ces entreprises considéraient le développement des capacités comme un coût, sans possibilité ou presque de tirer profit de ces capacités accrues, d'où la crainte de perdre ces salariés au profit d'autres employeurs. Dans les petites et moyennes entreprises, les contraintes résidaient dans des parcours professionnels internes limités qui empêchaient les salariés de progresser. Certains employeurs considéraient les salariés aspirant à développer leurs compétences et à progresser comme une source d'ennui.

De surcroît, le rôle accru des technologies dans certaines industries s'est traduit par une diminution du nombre de postes intéressants, et le désir de rentabilité à court terme des entreprises a abouti à une conception taylorienne ou fragmentée du travail. Cette situation a considérablement réduit la capabilité de ces salariés uniquement qualifiés dans des activités hyper spécialisées, et donc moins aptes à s'adapter aux changements. Il semble que certaines industries se trouvent dans un cercle vicieux: l'impossibilité de recruter pour des postes qualifiés entraîne une déqualification des processus et ainsi aucune génération future de travailleurs qualifiés n'est formée.

Enfin, nombre d'entretiens ont mis en évidence les freins au développement des capacités créés par d'autres personnes de l'organisation. Il s'agissait le plus souvent de dirigeants, supérieurs hiérarchiques ou collègues qui ne savaient pas comment partager leur savoir-faire et leurs connaissances avec les autres ou comment améliorer les capacités des autres, ou qui ne voulaient pas contribuer au développement des capacités des autres. De façon surprenante, en appliquant l'approche par les capacités, nous avons observé que la notion de capital humain et d'autres visions instrumentales des salariés et du développement prévalaient dans les entreprises.

Des freins institutionnels ont également été identifiés. Le principal frein se rapportait aux attitudes sociales (mentalités) généralement répandues dans la société néo-zélandaise qui ne valorisaient pas toujours l'éducation et décourageaient l'intérêt pour certaines professions. Les personnes interrogées associaient souvent ce frein aux changements de société. Dans les années 70, la Nouvelle-Zélande était surnommée le «half-gallon, quarter-acre, pavlova paradise» (d'après le titre du roman de Mitchell, 1972), un paradis où les familles vivaient dans des maisons individuelles sur leur lopin de terre (quarter acre) et où les enfants s'amusaient dehors à fabriquer des choses et à bricoler. Aujourd'hui, la population s'est urbanisée et les enfants ont des loisirs plus sédentaires tels que l'ordinateur, la télévision ou le shopping. Selon certaines personnes interrogées, ce changement de société est responsable du moindre intérêt des jeunes pour l'apprentissage et les métiers manuels. D'autres encore estimaient que l'école décourageait - ou n'encourageait pas - les étudiants à choisir certains métiers, professions et industries (faible mise en valeur des filières professionnelles, préjugés des parents, enseignants et conseillers d'orientation). Cette situation a été exacerbée par des tensions au sein de l'infrastructure de formation en entreprise et par la réticence des employeurs à prendre des apprentis/ stagiaires. Par exemple, dans les années 90, le système d'apprentissage était inexistant en Nouvelle-Zélande, et un cadre national de qualifications (NQF : National Qualifications Framework) a été mis en place pour valider la formation tout au long de la vie (des diplômes scolaires aux formations et qualifications postscolaires). Le NQF valide les formations conformément à des « unités » (unit standards) qui ont été enregistrés dans le NQF. De nombreuses unités ont été créées pour les différentes industries, professions et postes, en consultation avec les organisations de formation pour l'industrie (ITO = Industry Training Organisations) qui financent et gèrent la formation au sein des industries (par exemple, les ITO emploient les conseillers itinérants). Des salariés et des experts d'industrie ont évoqué certains problèmes posés par les unités utilisées dans l'industrie. Ces dernières sont perçues comme trop atomisées, découpant les processus en activités distinctes et séparées (chacune correspondant à un critère d'unité), mais sont inutiles sans une compréhension du processus global et de ses principes sous-jacents. Les personnes interrogées ont indiqué que les salariés formés selon ce système d'unités sont moins aptes à s'adapter aux changements des processus. C'est pourquoi les unités pourraient être interprétées comme un frein au développement des capacités. En fait, la politique de financement public des ITO a consisté à maximiser le 
nombre d'apprentis/stagiaires au moindre coût, sans se soucier véritablement de la qualité de la formation ou de ses résultats. Par ailleurs, des tensions ont été évoquées entre les ITO et les Instituts Polytechniques (établissements d'enseignement supérieur). Les Instituts Polytechniques sont financés en fonction du nombre d'inscrits et ils hésitent donc à développer ou dispenser de nouvelles formations professionnelles si un nombre minimum d'étudiants n'est pas garanti. Dès lors, l'enseignement et la formation professionnels n'ont pas toujours été capables de transmettre les compétences requises par la mutation de nombreuses industries.

La politique gouvernementale était jusqu'ici axée sur les compétences et leur validation dans une volonté de répondre aux préoccupations des employeurs concernant les pénuries de compétences dans certaines industries. Cependant, certaines personnes interrogées estimaient qu'il fallait adopter une approche plus holistique du développement des capacités au travail en Nouvelle-Zélande. Elles ont également souligné que le manque de coopération des employeurs (mauvaise volonté, absence d'opportunités) en matière de développement des capacités, pourrait constituer un frein permanent dans certaines industries.

Ainsi se dessine une image nette mais complexe des influences individuelles, organisationnelles et institutionnelles sur le développement des capacités dans les organisations néo-zélandaises étudiées.

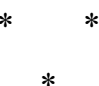

Sen, Nussbaum et d'autres reconnaissent l'interaction entre les individus et les institutions dans le développement et l'utilisation des capacités. Le travail joue un rôle important dans le développement des capacités. Il constitue à la fois un moteur et un frein. Nos recherches montrent que la réalité quotidienne du développement des capacités des salariés au sein d'organisations néo-zélandaises est influencée par de nombreux facteurs liés les uns aux autres: politique gouvernementale, pratiques de l'industrie et de l'éducation, comportement des employeurs, actions et mentalités des individus. Et cela renforce l'idée d'interactions dans la vie de l'individu - les expériences vécues dans la famille, dans la communauté, à l'école et au travail ont des répercussions sur le développement des capacités. Ces expériences façonnent les aspirations, la confiance en soi, la volonté de créer ou de saisir des opportunités afin de mener la vie à laquelle on aspire.

Les recherches montrent, comme le suggère également Sehnbruch (2004), que la qualité de l'emploi et du travail est un aspect important du développement des capacités. À savoir, la nature et la définition du travail ; les opportunités offertes ; l'environnement du travail et la qualité des relations avec les collègues et les supérieurs; le salaire et la reconnaissance. Mais, par-dessus tout, les recherches mettent en avant l'importance des encouragements qui peuvent se traduire par une plus grande confiance en soi, une meilleure estime de soi et, au final, une pro-activité de l'individu. En ce sens, la qualité de l'emploi permet d'atteindre l'une des «capacités fonctionnelles» fondamentales de Nussbaum: le contrôle de son environnement.

Les recherches révèlent également que le gouvernement et les employeurs considèrent majoritairement le développement des capacités dans les organisations comme un moyen d'atteindre les objectifs de production. Le développement d'autres capacités fonctionnelles est considéré comme un bonus. Cela est révélateur d'une approche complexe des compétences qui se reflète dans le dispositif de validation de la formation tout au long de la vie en Nouvelle-Zélande.

Cependant, il serait faux de croire que tous les individus aspirent à développer leurs capacités dans le cadre du travail ou dans quelque cadre formalisé que ce soit. Selon Sen (1993), la qualité de vie dépend de la capacité de l'individu à réaliser des fonctionnements auxquels il accorde de la valeur. Pour certains, le travail n'est qu'un moyen pour réaliser leurs fonctionnements de valeur ; pour d'autres, le travail représente leur fonctionnement de valeur (le travail est une fin en soi). Mais il serait également erroné de supposer que tous les individus ont suffisamment confiance en eux pour défendre les valeurs auxquelles ils croient ou qu'ils savent en fait à quoi ils aspirent.

Les gouvernements souhaitent que les citoyens apportent, d'une manière ou d'une autre, une contribution à la société. Beaucoup apportent cette contribution à 
travers le travail - un emploi rémunéré ou un travail non rémunéré dans la communauté ou au foyer. Le travail répond aux besoins sociaux, psychologiques, financiers et autres des individus. Ainsi, une expérience de travail est un ingrédient important dans le développement des capacités. Les nations, les mains-d'œuvre et les organisations «capables» s'appuient sur des individus «capables » et sur des communautés, des politiques, des systèmes, des infrastructures et des ressources auxquels ces individus s'adaptent ou qu'ils créent ou modifient. Dans ces recherches, nous nous sommes concentré sur le lieu de travail organisationnel et nous pouvons nous interroger sur ce qu'il est raisonnable d'attendre des employeurs en matière de développement des capacités.

Nous pouvons ainsi raisonnablement attendre des employeurs qu'ils s'engagent à investir dans le développement des capacités de leurs salariés. Par exemple, au travers de divers mécanismes tels que la formation, une meilleure définition du travail, des compétences managériales et d'encadrement améliorées, une attitude de soutien et d'encouragement contribuant à une meilleure estime de soi.

Un accord entre les citoyens, le gouvernement et les entreprises - un partenariat social relatif au travail et au développement des capacités - est-il nécessaire ? Le problème, c'est que pour gagner du temps, la plupart des gouvernements consultent uniquement les principales associations patronales et syndicales. Pourtant, ces groupes (notamment en NouvelleZélande) sont rarement représentatifs de la multitude de petites organisations, comme celles étudiées dans les recherches présentées dans cet article. De ce fait, les résultats de ces consultations ont peu d'impact sur ces organisations qui ne se sentent pas véritablement concernées. Ainsi, le défi majeur auquel sont confrontés les gouvernements engagés dans une démarche de développement des capacités au travail est de parvenir à atteindre et à impliquer ces organisations petites ou moyennes.

Traduction : TSF

\section{Biobliographie}

Basu K. \& Lopez-Calva L.F.(2002), "Functionings and capabilities", in K. Arrow A. Sen. \& K. Suzumura (eds), Handbook of social choice and welfare, London: Elsevier.

Becker B. \& Gerhart B. (1996), "The impact of human resource management on organizational performance: progress and prospects", Academy of Management Journal, 39 (4), pp. 779-801.

Blackwood L., Feinberg-Danieli G., Lafferty G. \& Kiely P. (2005), Employment agreements: Bargaining trends \& Employment law 2004/2005, Wellington: Industrial Relations Centre, Victoria University.

Brown R. \& McCartney S. (2004), “The development of capability: the content of potential and the potential of content", Education and Training, 46 (1), pp. 7-10.
Budd J. W. (2004), Employment with a human face, Ithaca: Cornell University Press.

Burchardt T. (2002), Constraint and opportunity: women's employment in Britain. Paper presented at the Von Hugel Institute Conference - Promoting Women's Capabilities: examining Nussbaum's capabilities appproach, septembre 2002, Cambridge.

Deeks J. \& Rasmussen E. (2002), Employment relations in New Zealand. Auckland, N.Z: Pearson Education Limited New Zealand.

Fenwick T. (1998), "Questioning the concept of the learning organisation", in S. Scott, B. Spencer, \& A.Thomas (Eds.), Learning for life, Toronto: Thompson Educational Publishing, pp.140-152. 
Huselid M. (1995), “The impact of human resource management practices on turnover, productivity and corporate financial performance", Academy of Management Journal, 38(3), pp. 635-672.

King N. (Ed.) (1998), Template analysis, London: Sage.

Kvale S. (1996), Interviews: An introduction to qualitative research interviewing, Thousand Oaks, C.A: Sage Publications.

Matthews P. (1999), "Workplace learning: Developing an holistic model", The Learning Organization, 6(1), pp. 18-29.

Ministry of Economic Development (2003), SME's in New Zealand: structure and dynamics, Wellington, New Zealand: Author.

Mitchell A. (1972), The Half-gallon Quarter-acre Pavlova Paradise, Whitcombe \& Tombes: NZ

Mojab S. \& Gorman R. (2003), "Women and Consciousness in the "Learning Organization": Emancipation or Exploitation?" Adult Education Quarterly, 53(4), pp. 228-241.

Nussbaum M. (1999), "Women and equality: The capabilities approach", International Labour Review, 3, pp. 227-245.

Patton M. Q. (2002), Qualitative evaluation and research methods (3rd ed.), London: Sage Publications.

Peirce J. (2000), Canadian Industrial Relations, Scarborough, Ontario: Prentice-Hall Canada Inc.

Pfeffer J. (1994), Competitive advantage through people, Boston: Harvard Business School Press.
Salais R., Villeneuve R. (sous la dir.) (2006), Développer les capacités des hommes et des territories en Europe, (traduction de l'ouvrage paru aux presses universitaires de Cambridge), Éditions de l'Anact, $452 \mathrm{p}$.

Sehnbruch K. (2004), From the quantity of employment to the quality of employment: An application of the capability approach to the Chilean Labour Market. California, USA: University of California.

Sehnbruch K. (2005), The capability approach: Just the latest fashionable trend in economic thought?, Retrieved from: http//tere.r-t.cl/ei/paper 11.pdf.

Sen A. (1993), "Capability and Wellbeing", in M. Nussbaum \& A. Sen (Eds.), The Quality of Life (pp. 30-53), Oxford: Oxford University Press.

Sen A. (1997) [1959-1961], "Editorial: Human capital and human capability", World Development, 34 (3).

Sen A. (1999), Development as Freedom, Oxford: Oxford University Press.

Senge P. (1990), The Fifth Discipline: the art and practice of the learning organisation, New York: Doubleday.

Stake R. (1995), The art of case study research, London: Sage Publications.

Thomson A., Mabey C., Storey J., Gray C. \& Iles P. (2001), Changing patterns of management development, Oxford: Blackwell.

Yin R. K. (2003), Case study research: Design and methods (3rd ed.), Thousand Oaks, C.A: Sage Publications. 


\title{
Résumé
}

\section{Le travail et le développement des capacités}

\author{
Par Jane Bryson et Kiri Merritt
}

Cet article explore les moteurs, les éléments contributifs et les freins au développement des capacités au travail. II passe en revue les principales thématiques de la littérature consacrée aux capabilités $\left({ }^{*}\right)$ et met en évidence les tensions entre les visions utilitaristes étroites de la théorie du capital humain et I'approche par les capacités plus holistique défendue par Amartya Sen. Cet article présente ensuite des travaux de recherche qui étudient les influences institutionnelles, organisationnelles et individuelles sur le développement des capacités dans des organisations néo-zélandaises. II analyse enfin le rôle du travail dans le développement des capacités et met en lumière des facteurs importants tels que la qualité de l'emploi et l'influence de personnes clés sur les progrès individuels. Et il conclut sur la question suivante: que peut-on raisonnablement attendre des gouvernements et des employeurs en matière de contribution au développement des capacités?

${ }^{*}{ }^{*}$ Le mot anglais " capability » est traduit par le néologisme "capabilité » ou, par souci de simplicité, par " capacité » dans les expressions « approche par les capacités » et " développement des capacités ».

Mots-clés

Approche par les capabilités, théorie du capital humain, travail, Nouvelle-Zélande

Journal of Economic Literature: D 63, J 24, J 59 